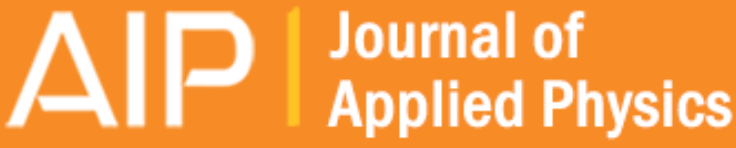

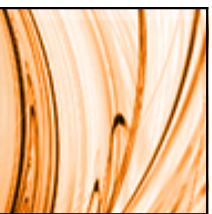

Anomaly close to an electronic topological semimetal-insulator transition in elemental fcc-Yb under pressure

Carsten Enderlein, Scheilla M. Ramos, Magda Bittencourt, Mucio A. Continentino, William Brewer, and Elisa

Baggio-Saitovich

Citation: Journal of Applied Physics 114, 143711 (2013); doi: 10.1063/1.4825073

View online: http://dx.doi.org/10.1063/1.4825073

View Table of Contents: http://scitation.aip.org/content/aip/journal/jap/114/14?ver=pdfcov

Published by the AIP Publishing

\section{Articles you may be interested in}

Semiconductor-to-metal transition of $\mathrm{Bi2Se} 3$ under high pressure

Appl. Phys. Lett. 105, 062102 (2014); 10.1063/1.4892661

Electronic topological transition and semiconductor-to-metal conversion of $\mathrm{Bi} 2 \mathrm{Te} 3$ under high pressure Appl. Phys. Lett. 103, 052102 (2013); 10.1063/1.4816758

Effect of uniaxial pressure on metal-insulator transition in ( $\mathrm{Sm} 1$ y Nd y ) $0.52 \mathrm{Sr} 0.48 \mathrm{MnO} 3$ single crystals Appl. Phys. Lett. 94, 252506 (2009); 10.1063/1.3160019

Study on Electronic States of Solids Under High Pressure by Infrared Synchrotron Radiation AIP Conf. Proc. 902, 71 (2007); 10.1063/1.2723625

Dynamics of the insulator-conductor transition initiated by high pressure in ammonium halides Low Temp. Phys. 30, 916 (2004); 10.1063/1.1820024

AIP $\left.\right|_{\text {Letters }} ^{\text {Applied Physics }}$

is pleased to announce Reuben Collins as its new Editor-in-Chief

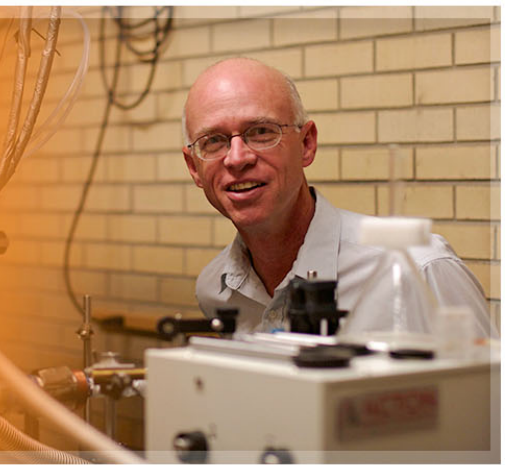




\title{
Anomaly close to an electronic topological semimetal-insulator transition in elemental fcc-Yb under pressure
}

\author{
Carsten Enderlein, ${ }^{1}$ Scheilla M. Ramos, ${ }^{2}$ Magda Bittencourt, ${ }^{1}$ Mucio A. Continentino, ${ }^{1}$ \\ William Brewer, ${ }^{3}$ and Elisa Baggio-Saitovich ${ }^{1}$ \\ ${ }^{1}$ Centro Brasileiro de Pesquisas físicas, CBPF, Rua Dr. Xavier Sigaud 150, Rio de Janeiro, Brazil \\ ${ }^{2}$ Département de Recherche Fondamentale sur la Matiére Condensée, SPSMS, CEA-Grenoble, \\ 17 rue des Martyrs, Grenoble, France \\ ${ }^{3}$ Fachbereich Physik, Freie Universität Berlin, Arnimallee 14, Berlin, Germany
}

(Received 31 July 2013; accepted 27 September 2013; published online 11 October 2013)

\begin{abstract}
The Lifshitz-type semimetal-insulator transition, which is a transition of the electronic topology, has been considered as the most fundamental metal-insulator transition. Here, we present resistivity measurements under pressure in the vicinity of the quantum critical point of fcc $\mathrm{Yb}$. We apply a previously suggested scaling for this type of transition and identify its universality class. Moreover, we observe an anomaly in the screening coefficient $A$ of the $T^{2}$ term in the resistivity at low temperatures in the metallic phase. We suggest an interpretation of this phenomenon as an effect of doping by $\mathrm{Ca}$ impurities unintentionally present in the $\mathrm{Yb}$ crystals. The observed behavior may very well be applicable to any doped system in the vicinity of such a transition. (C) 2013 AIP Publishing LLC. [http://dx.doi.org/10.1063/1.4825073]
\end{abstract}

\section{INTRODUCTION}

An electronic topological continuous phase transition (often referred to as a "Lifshitz transition") corresponds to a drastic change of the Fermi surface. This can be for instance the vanishing or the appearance of an electron or hole pocket. ${ }^{1}$ Clearly, if each and every electron and hole pocket of a semimetal vanishes and a gapped state remains, the material undergoes a transition from a semimetallic compressible quantum-matter state to a trivially gapped state of the BlochWilson type. ${ }^{2}$ This type of metal-insulator transition (MIT) - a so-called "Wilson transition"- - has been considered to be the simplest and most fundamental type of MIT, since no chemical changes, magnetic phenomena or other concomitant phenomena are involved. ${ }^{3}$ However, probably due to its apparent simplicity, in most review papers on MITs the Wilson transition is discussed only briefly. ${ }^{4,5}$ Recently, there has been renewed interest in this topic, due to the implied unusual quantum criticality of Lifshitz transitions, ${ }^{6}$ and also due to new experimental evidence for the existence of possible quasi-particle interactions that avoid such Wilson transitions. ${ }^{7,8}$

The pressure-induced MIT in fcc Ytterbium was supposed early on to offer a perfect example of a Wilson transition. 9 The material becomes insulating under modest pressures of about $15 \mathrm{kbars},{ }^{10,11}$ most likely due to a hybridization of the valence s-p bands with the empty d states above the Fermi level. ${ }^{12}$ The universality of such a transition has been described by Continentino. ${ }^{13,14}$ In addition to our results on the effects of slight impurity doping on Lifshitz transitions, this paper also gives - to our knowledge the first - application of the underlying scaling theory to these transitions.

\section{EXPERIMENTS}

Here, we present temperature-dependent measurements of the AC resistivity of bulk ytterbium under varying hydrostatic pressures. The fcc Yb sample was purchased from Alfa
Aesar and has a nominal purity of $99.9 \%$, with about $0.1 \%$ $\mathrm{Ca}$ impurities. Our measurements cover the pressure range from 1 bar to $30 \mathrm{kbars}$ and the temperature range from 1.4 to $300 \mathrm{~K}$. Measurements were performed using a 4-point probe with an AC resistance bridge (LR-700). The sample was installed in a standard piston-cylinder pressure cell with a mixture of Fluorinert F77 and F70 as the pressure transmitting medium. The pressure was applied while measuring the resistance of a manganin wire to gain a rough measure of the applied pressure. During the measurements, the superconducting transition of a lead sample inside the cell was determined to provide a precise value of the applied pressure at low temperatures. The low temperatures were obtained using a ${ }^{4}$ He flow cryostat.

The measurements were performed without stabilizing the temperature at each measurement point. Instead, measurement points were recorded at a cooling rate of around $3 \mathrm{~K}$ per hour in the critical regions (i.e., at low temperatures). If the cooling rate was poorly controlled or anomalies were observed, a second measurement was performed by warming up the cold sample slowly and comparing the resistivity curve from the cooling with that from the warming process.

\section{RESULTS}

An overview of the measurements is presented in Fig. 1(a). R-T-curves were determined at 25 different pressures between 0 and 30 kbars. As can readily be seen from the figure, resistivity measurements show, as expected, a positive $d \rho / d T$ in the metallic phase. A quantitative analysis reveals that the resistivity in the metallic phase at temperatures below $15 \mathrm{~K}$ can be well described by $\rho(T)=\rho_{0}+A T^{2}+B T^{5}$. At $1 \mathrm{~K}$, the value of $B \cdot T^{3}$ is about three orders of magnitude smaller than $A$, and it is of the same order of magnitude at $10 \mathrm{~K}$. A nearly pure $T^{2}$ dependence can be observed up to $8 \mathrm{~K}$ (see the fits in the appendix). The temperature below which the function 

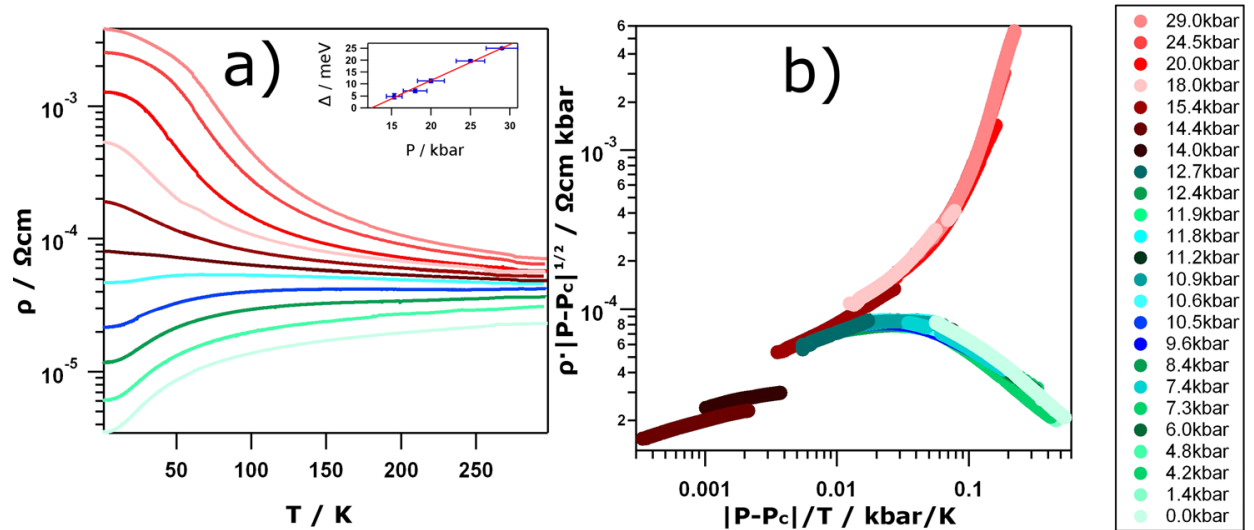

FIG. 1. Resistivity measurements and the corresponding scaling for critical exponents $\nu=1 / 2, \nu z=1$, and $P_{c}=14.3$ kbars. (a) For clarity only, selected resistivity curves are shown in the left panel. Different pressures correspond to different colors as indicated by the legend on the right side. Colors were chosen in such a way that red-colored curves correspond to measurements in the semiconducting phase and blue/green-colored curves correspond to those in the metallic phase. The inset shows the gap size as a function of the pressure. The data points were extracted from the exponential slope at higher temperatures in the insulating phase. (b) All measured data (including the data, which has not been shown in the left panel) in the scaling form represented by Eq. (1), with their respective critical exponents. All of the measured resistivity curves are included in this figure.

gives a good fit remains constant over the pressure range from 0 to $12 \mathrm{kbars}$, as can be seen in Fig. 2(d).

The $T^{5}$ dependence is a necessary feature that is directly related to the phonon contribution to the resistivity, while $\rho_{0}$ is the residual resistivity, which is generally attributed to impurities and defects in the metallic crystal. The $T^{2}$ dependence at low temperatures in metals is often attributed to Fermi liquid behavior. However, the low value of $A$ and the fact that $T_{A}$, as indicated in Fig. 2(d), remains constant, contradict such an interpretation. This will be further discussed below in Sec. IV, where we suggest that Taylor-Koshino scattering ${ }^{16}$ is responsible for the observed behavior. In Figs. 2(a) and 2(b), the pressure dependencies of $A$ and $\rho_{0}$ are shown. A strong kink is observed in $A$ at 10 kbars. Our data also show a kink-like feature in $\rho_{0}$ at the same pressure, as can be seen more clearly in the $\rho_{0} v s . P^{-1 / 2}$ plot given as the inset in Fig. 2(b). The $P^{-1 / 2}$ scaling follows from simple scaling laws close to a Lifshitz transition. ${ }^{13}$ Above 10 kbars, $A$ decreases rather rapidly with increasing pressure. Measurements at pressures above 12 kbars show strong deviations from $T^{2}$ behavior and do not permit us to determine $\rho_{0}$ reliably.

Above $15 \mathrm{~K}$, all curves up to 10(1) kbars show generally positive $d \rho / d T$, and the insulating phase exhibits the expected negative $d \rho / d T$ behavior over the whole temperature range. Above 10 kbars, the resistivity slope at temperatures above $80 \mathrm{~K}$ becomes negative. Below this temperature, the slope remains positive. Above 14 kbars, the system seems to be a semiconductor. It should be pointed out that our measurements do not reveal any resistivity divergence


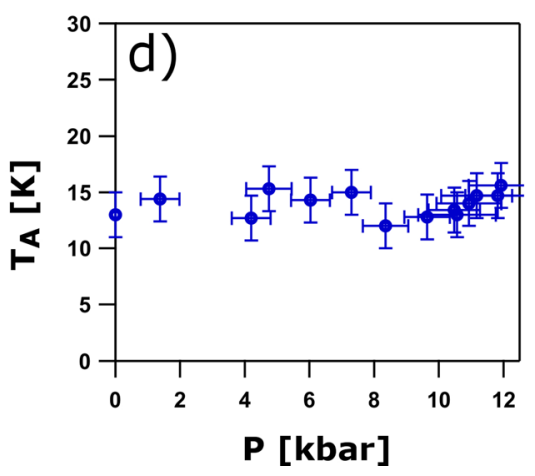

FIG. 2. The pressure dependence of essential metallic constants: (a) The pressure dependence of the coefficient $A$ as determined from a fit to the resistivity of the form $\rho=\rho_{0}+A T^{2}+B T^{5}$. It can be clearly seen that $A$ peaks near 10 kbars. (b) $\rho_{0}$ increases over the whole critical region and becomes indeterminate around 12 kbars. Inset: $\rho_{0}$ vs. $P^{-1 / 2}$. In agreement with the theory of Lifshitz transitions, the resistivity scales as $P^{-1 / 2} \cdot{ }^{14}$ A kink at a value corresponding to 10 (1) kbars is clearly visible. This would be expected for any Lifshitz transition. ${ }^{15}$ (c) $A$ as a function of $\rho_{0}$. Up to $10 \mathrm{kbars}$, it shows a positive linear behavior. Above 10 kbars, $A$ decreases. (d) The temperature below which the $T^{2}$ fit can be applied remains constant over the whole pressure range in the metallic phase. 
on approaching $0 \mathrm{~K}$, as would be expected for a clean insulator. Instead, the resistivity shows a saturation, and at the highest pressure measured, a slight slope is seen, indicating a possible resistivity divergence on approaching $0 \mathrm{~K}$ (not shown in the paper). This will be discussed further in Sec. IV. At pressures above 18 kbars, the curves show a clear $\ln \rho=-\Delta / k_{B} T$ behavior for temperatures above the saturation range, as generally expected for semiconductors. ${ }^{17}$ In the inset of Fig. 1(a), the opening of the gap as a function of pressure is exhibited; a linear extrapolation leads to a pressure corresponding to a zero gap at 12(2) kbars. Such a linear extrapolation is reasonable since the gap size should scale linearly with pressure for such a transition. ${ }^{13} 12(2)$ kbars is rather close to the point where $A$ vanishes, as mentioned above.

According to Continentino, ${ }^{13,14,18}$ the scaling law for the conductivity of a 2 nd order MIT is

$$
\sigma=\xi^{(2-d)} f\left(\frac{\left(P_{c}-P\right)^{\nu z}}{T}\right),
$$

where $d$ is the dimension of the system, which is naturally 3 in our case. The correlation length $\xi$ can be estimated to be proportional to $\left|P-P_{c}\right|^{-\nu}$. Universality of this transition is associated with the critical exponents $\nu=1 / 2$ and $\nu z=1$. Furthermore, these exponents are related to the dimensionality of the system by the quantum hyperscaling relation $2-\alpha=\nu(d+z)$, where $2-\alpha$ is the critical exponent for the heat capacity. In three dimensions, this yields $2-\alpha=2.5$, a value also used to designate the Lifshitz transition. ${ }^{13,14}$ The scaling is visualized in Fig. 2(b) by plotting $\rho \cdot \sqrt{\left|P_{c}-P\right|} v s$. $\left|P_{c}-P\right| / T$, with $P_{c}=14.3$ kbars. It does not apply at very low temperatures, where the $T^{2}$ behavior in the metallic phase sets in. In the insulating phase, the scaling does not give a good fit in the saturation range. The corresponding temperature regions have been suppressed in the scaling plot of Fig. 1(b). For the remaining data points, the scaling is excellent using $P_{c}=14.3(2) \mathrm{kbars}$, and we interpret this pressure as the critical pressure of the MIT, above which the system becomes strictly insulating. This scaling plot allows us to extract the asymptotic form of the scaling, which makes it possible to determine the value of $2-\alpha$ to be $2.4 \pm 0.1$. This is in good agreement with the expected value of 2.5.

\section{DISCUSSION}

In a nutshell, we make the following observations that are worthy of further discussion:

- At 10(1) kbars, we observe a kink in $A$, which is accompanied by a kink in $\rho_{0}$.

- The linear extrapolation of the gap size from resistivity measurements in the insulating phase leads to a zero-gap pressure of 12(2) kbars.

- The scaling gives a critical pressure for the metalinsulator transition of 14.3(2) kbars.

- The temperature below which the resistivity can be fitted by $\rho(T)=\rho_{0}+A T^{2}+B T^{5}$ remains constant from 0 to 12 kbars.

- The resistivity seems to saturate when approaching low temperatures in the presumably semiconducting phase, and shows only a slight upward turn for the 29 kbars curve at around $4 \mathrm{~K}$.

Before giving a further discussion concerning the MIT, we should mention that theory and experiment agree that there is an MIT of the Lifshitz type in this material, ${ }^{3,10,12,18-20}$ and our resistivity curves agree very well with previous work on the same material, ${ }^{11}$ which makes it very unlikely that our observed system is not fcc $\mathrm{Yb}$ and/or does not undergo the MIT as proposed.

Our data interpretation leads us to believe that the gapopening pressure corresponds to 12(2) kbars, as established from the fit of the gap size. It should be mentioned thatalthough this value agrees roughly with the measured value for the MIT of 14.3(2) kbar established from the scaling within quoted errors - the fact that the two values have been extracted from exactly the same data can be seen as an indication that these two values are indeed different. The same consideration applies to the pressure value for the kink in $A$, which, at 10(1) kbars, is rather far from $14.3 \mathrm{kbars}$, but also does not likely correspond to the gap opening.

We hold that the above observations can be well explained by assuming that the $\mathrm{Ca}$ impurities present in our $\mathrm{Yb}$ crystal dope the material. As will be discussed below, this assumption is consistent with all of the observations mentioned above.

To visualize the effect of Ca-impurities on a Wilson transition, we start with a thought experiment. We imagine a pure, defect- and disorder-free crystal with a vanishing indirect band gap at the Fermi level (zero-gap semiconductor). As expected for ytterbium, we further assume only one valence and one conduction band. If impurities which could serve as donors or acceptors are introduced into this intrinsic system, the energy level of impurity-related holes or electrons must lie in a region of the continuous energy spectrum. ${ }^{21}$ Naturally in such a system, we expect an extremely low density of states at the Fermi level. Therefore, a small number of donors can be expected to show a strong effect. The resulting three different steps from a semimetal to a semiconductor with impurities are shown in Fig. 3. Impurity doping will lead to a shift of the Fermi level. Approaching from the semiconducting side, the sample must become metallic when the band gap is smaller than the impurity energy level. The consequence is a strong doping sensitivity of the underlying system. Therefore, the transition will occur in steps as demonstrated in Fig. 3. Since the Yb crystal contains



FIG. 3. Schematic of the four electronic configurations of the metalinsulator transition as proposed. The pure material is a doped semimetal. At 10 kbars, the van Hove singularity of the lower band passes through the Fermi level, leading to a Lifshitz transition of order 2.5. At $12 \mathrm{kbars}$, the opening of the gap approaches 0 . The system does not undergo a phase transition at this point. The metal-insulator transition occurs when the other van Hove singularity passes through the Fermi level. 
mainly $\mathrm{Ca}$ impurities, we suggest that these impurities create the situation described.

A natural question that arises is where the energy levels of these impurities lie. The slope that is shown in the inset of Fig. 1(b) suggests that their energies lie in the meV range. The facts that the gap opens with roughly $1.5 \mathrm{meV} / \mathrm{kbar}$, and about 2 kbars lie between the opening of the gap and the last Lifshitz transition supports this. However, these are very rough values, since it is likely that the permittivity strongly changes during the transition, which would make the impurity energy levels pressure dependent.

In the following, we will discuss the $T^{2}$ behavior of the $\mathrm{Yb}$ resistivity in the metallic phase. Mechanisms which can cause a $T^{2}$ dependence of the resistivity at low temperatures include electron-electron scattering, electron-hole scattering, ${ }^{22}$ and impurity-phonon scattering ${ }^{16}$ (along with others ${ }^{23}$ ). In previous work, the $T^{2}$ behavior in $\mathrm{Yb}$ close to the Wilson transition has been attributed to electron-hole scattering of the Baber type., ${ }^{3,10,11,22}$ However, Baber scattering contradicts observed phenomena. We argue that the measured $T^{2}$ behavior might in fact originate from inelastic scattering on impurity phonons (Taylor-Koshino scattering). ${ }^{16}$ The explanation via Baber scattering is particularly inappropriate since scaling theory predicts a vanishing coherence temperature in the vicinity of the transition $\left(T_{c o h} \propto \Delta^{\nu z}\right){ }^{4}$ The coherence temperature can naturally be determined from the temperature below which the $T^{2}$ behavior still persists. Our measurements reveal this temperature to be independent of pressure, as demonstrated in Fig. 2(d). This is a strong sign that the origin of the behavior lies in a process that is not affected by the approach to the MIT. Moreover, for electron-electron scattering, $A$ should diverge when approaching the MIT. Instead, it peaks at $10 \mathrm{kbars}$ and then drops monotonically to $12 \mathrm{kbars}$.

The $T^{2}$ coefficient $A$ shows a linear dependence on $\rho_{0}$ up to the first Lifshitz transition at 10 kbars (Fig. 2(c)). This is a typical signature of Taylor-Koshino scattering. However, the number and the error bars of our data points do not allow a final decision about the proportionality of the two variables. One might think that the fact that $A$ drops sharply contradicts the hypothesis of an impurity-phonon scattering mechanism. Therefore, it should be pointed out that the number of charge carriers should drop strongly after the first Lifshitz transition. This will greatly influence scattering mechanisms, and we are not aware of any calculations of impurity-induced scattering mechanisms in the direct vicinity of a Wilson transition. Therefore, we leave the final interpretation of the $T^{2}$ behavior open, but suggest Taylor-Koshino scattering as the most satisfactory way to explain this behavior. This interpretation is quite consistent with the other observed impurity-related effects.

As previously mentioned, the resistivity in the low temperature range does not obey the scaling law. This is in agreement with impurity-induced effects, as the Wilson transition is a transition of the topology of the Fermi surface and a disordered system cannot exhibit such a transition. This suggests that in the low-temperature regime, not only in the metallic phase, but also in the semiconducting phase, the resistivity behavior is dominated by impurities. Therefore, the small band-gap semiconducting system is an extrinsic semiconductor. This explains the saturation range of the resistivity in the insulating phase. Impurity-induced broadening of the bands, as discussed theoretically by Blanter and collaborators, ${ }^{15}$ can be neglected, since its effects are too weak to be apparent in our data.

A Lifshitz transition should be accompanied by a kink in the resistivity, ${ }^{15}$ as we have observed, compare Fig. 2(b). However, if it is accompanied by a metal-insulator transition, the residual conductivity should be zero and therefore the residual resistivity infinite. Accordingly, we interpret our results in such way that the $\mathrm{Ca}$ impurities induce doping as demonstrated in Fig. 3.

Since the three-step process, as exemplified in Fig. 3, is clearly more complicated than a simple Wilson transition, we want to refer to the points discussed above to compare the two scenarios:

- The kink in A at 10 kbars (Fig. 2(a)): Assuming a single Wilson transition, it would be very hard to find an explanation for the kink or for any rapid change in the slope of the $A$ vs. $P$ curve. However, a kink could be explained naturally by the three-step process, as discussed above.

- The $\rho_{0}$ vs. $P$ curve: A weak kink in the resistivity would be expected in the case of a Lifshitz transition; ${ }^{15}$ since the resistivity should scale with the critical exponent $-\nu=-1 / 2$, the kink becomes clearly visible in the $\rho_{0}$ vs. $P^{-1 / 2}$ curve. This observed kink might be difficult to explain within the model of a single Wilson transition.

- The saturation of the resistivity on approaching low temperatures: This phenomenon would be very puzzling when considering an undoped crystal (as would be necessary for a single Wilson transition). Since the existence of the MIT is well established, it seems necessary to interpret these curves as belonging to an extrinsic semiconductor. This, however, establishes the three-step process, as explained above.

- The difference between the pressure for the gap opening (12(2) kbars) and the critical pressure, as calculated from scaling (14.3(2) kbars): Considering simply the error bars, it might seem possible that these two analyses actually give the same value, although this is not very probable. This interpretation is, however, unlikely, since the two values were extracted from the same data. An attempt to use $12 \mathrm{kbars}$ as the critical pressure for the scaling does not yield acceptable results.

\section{v. CONCLUSIONS}

We have performed temperature- and pressuredependent resistivity measurements on fcc bulk ytterbium. Our measurements reveal the opening of a band gap at approximately 12 kbars. This gap opening is not directly accompanied by a Lifshitz transition, since the transitions of the pocket-vanishing type appear before and after this gap opening, namely at around 10(2) and 14.3(2) kbars. The MIT that accompanies the transition at 14.3 kbars can be perfectly scaled with the approach of Continentino for Wilson transitions. ${ }^{13}$

To our present knowledge, this work, which represents the observation of a pure Wilson transition, is the first to show an MIT with a scaling which completely neglects 


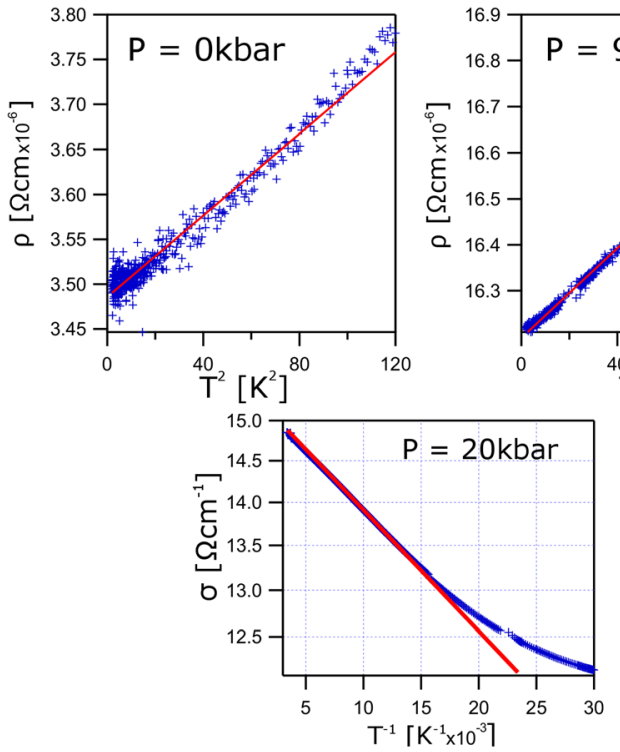

electron-electron interactions. Moreover, we have shown the effect of impurities on the transport behavior of the observed system. This implies the $T^{2}$ behavior of the resistivity, previously interpreted as due to Baber scattering at low temperatures in the metallic phase, as well as the resistivity saturation in the semiconducting phase and a doping-induced splitting of the pressure-degenerate quantum criticality.

\section{ACKNOWLEDGMENTS}

This project was financially supported by MCT, CNPq, and FAPERJ. We thank E. N. Hering for help with the experiments and fruitful discussions.

\section{APPENDIX: FITS APPLIED}

Representative fits of the low-temperature regime in the metallic range are shown in the upper row of Fig. 4 . The fits for the evaluation of the data were obtained in a straightforward manner by fitting the function $a+b T^{2}+c T^{5}$ using the IGOR Pro curve fitting tool to the original curves. The images in the upper row of Fig. 4 all give $\rho v s . T^{2}$ (neglecting the $T^{5}$ term), since it is easier to see the $T^{2}$ behavior with the unaided eye in this way.

The lower row in Fig. 4 shows the fits in the intrinsic semiconducting region. Deviations in the low-temperature range appear naturally when impurity doping is present. The slight deviations at higher temperatures are very probably due to measurement errors, since close to room temperature the temperature control during the measurements was less reliable.

${ }^{1}$ I. M. Lifshitz, "Anomalies of electron characteristics of a metal in the high pressure region," Sovi. Phys. JETP 11, 1130-1135 (1960).

${ }^{2} \mathrm{G}$. E. Volovik, Quantum Phase Transitions from Topology in Momentum Space in Quantum Analogues: From Phase Transitions to Black Holes and Cosmology (Springer, Berlin, 2007).

${ }^{3}$ N. F. Mott and Z Zinamon, "The metal-nonmetal transition," Rep. Progr. Phys. 33, 881-940 (1970).

${ }^{4}$ M. Imada, A. Fujimori, and Y. Tokura, "Metal-insulator transitions," Rev. Mod. Phys. 70, 1039-1263 (1998).

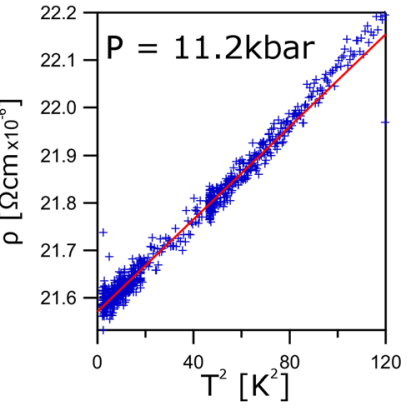

FIG. 4. Upper row: $\rho$ vs. $T^{2}$ for three representative pressures. Lower row: Two representative fits for the intrinsic semiconducting region.
${ }^{5}$ P. P. Edwards, R. L. Johnston, C. N. R. Rao, D. P. Tunstall, and F. Hensel, "The metal-insulator transition: a perspective," Philos. Trans. R. Soc. 356, 5-22 (1998).

${ }^{6} \mathrm{M}$. Imada, T. Misawa, and Y. Yamaji, "Unconventional quantum criticality emerging as a new common language of transition-metal compunds, heavy-fermion systems, and organic conductors," J. Phys.: Condens. Matter 22, 164206(9pp) (2010).

${ }^{7}$ N. P. Armitage, R. Tediosi, F. Lvy, E. Giannini, L. Forro, and D. van der Marel, "Infrared conductivity of elemental bismuth under pressure: Evidence for an avoided Lifshitz-type semimetal-semiconductor transistion," Phys. Rev. Lett. 104, 237401(4pp) (2010).

${ }^{8}$ H. Cercellier, C. Monney, F. Clerc, C. Battaglia, L. Despont, M. G. Garnier, H. Beck, and P. Aebi, "Evidence for an excitonic insulator phase in 1T-TiSe2," Phys. Rev. Lett. 99, 146403(4pp) (2007).

${ }^{9}$ N. F. Mott, "The transition to the metallic state," Philos. Mag. 6, 287-309 (1961).

${ }^{10}$ D. B. McWhan, T. M. Rice, and P. H. Schmidt, "Metal-semiconductor transition in ytterbium and strontium at high pressures," Phys. Rev. 177, 1063-1071 (1969).

${ }^{11} \mathrm{R}$. Jullien and D. Jerome, "Etude de l'ytterbium et des alliages ytterbiumbaryum sous haute pression a basse temperature," J. Phys. Chem. Solids 32, 257-265 (1971).

${ }^{12}$ G. Johansen and A. R. Mackintosh, "Electronic and phase transitions in ytterbium," Solid State Commun. 8, 121-124 (1970).

${ }^{13}$ M. A. Continentino, "Metal-insulator transition in semi-metals and kondo insulators," Phys. Lett. A 197, 417-422 (1995).

${ }^{14}$ M. A. Continentino, Quantum Scaling in Many-Body Systems (World Scientific, 2001).

${ }^{15}$ Y. M. Blanter, M. I. Kaganov, A. V. Pantsulaya, and A. A. Varlamov, "The theory of electronic topological transitions," Phys. Rep. 245, 159-25 (1994).

${ }^{16}$ P. L. Taylor, "The inelastic scattering of electrons at impurities in metals," Process Phys. Soc. 80, 755-758 (1962).

${ }^{17}$ N. W. Ashcroft and N. D. Mermin, Solid State Physics (Brooks/Cole, Belmont, 1976).

${ }^{18}$ M. A. Continentino, B. Elschner, and G. Jakob, "Metal-insulator transition in ytterbium under pressure: an EPR study," Europhys. Lett. 31, 485-490 (1995).

${ }^{19} \mathrm{Y}$. Kubo, "Self-consistent relativistic band structures of $\mathrm{Sr}$ and $\mathrm{Yb}$ under normal and high pressure," J. Phys. F: Met. Phys. 17, 383-396 (1987).

${ }^{20}$ R. H. Mutlu, "Calculated high-pressure-induced electronic and structural phase transitions in Sr and $\mathrm{Yb}$ up to 50 kbar," Phys. Rev. B 54, 16321 (1996).

${ }^{21}$ B. L. Gel'mont, V. I. Ivanov-Omskii, and I. M. Tsidil'kovskii, "The electronic energy spectrum of zero-gap semiconductors," Sov. Phys. Usp. 19, 879 (1976).

${ }^{22} \mathrm{D}$. Jerome, M. Rieux, and J. Friedel, "Low-temperatures resistivity in nearly excitonic systems," Philos. Mag. 23, 1061-1075 (1971).

${ }^{23}$ G. D. Mahan, Many-Particle Physics (Kluwer Academic/Plenum Publisher, New York, 1981). 